\title{
Transapical miniaturized ventricular assist device: Design and initial testing
}

\author{
Mark S. Slaughter, MD, ${ }^{\mathrm{a}}$ Guruprasad A. Giridharan, $\mathrm{PhD},{ }^{\mathrm{b}}$ Dan Tamez, BS, ${ }^{\mathrm{d}}$ Jeff LaRose, MS, ${ }^{\mathrm{d}}$ \\ Mike A. Sobieski, RN, CCP, ${ }^{\mathrm{a}}$ Leslie Sherwood, DVM, ${ }^{\mathrm{c}}$ and Steven C. Koenig, PhD ${ }^{\mathrm{a}, \mathrm{b}}$
}

Background: Left ventricular assist devices are increasingly used to treat patients with advanced and otherwise refractory heart failure as bridge to transplant or destination therapy. We evaluated a new miniaturized left ventricular assist device that requires minimal surgery for implantation, potentially allowing implantation in earlier stage heart failure.

\begin{abstract}
Methods: HeartWare (Miami Lakes, Fla) developed transapical miniaturized ventricular assist device. Acute $(\mathrm{n}=4)$, 1-week $(\mathrm{n}=2)$, and 30-day $(\mathrm{n}=4)$ bovine model experiments evaluated hemodynamic efficacy and biocompatibility of the device, which was implanted through small left thoracotomy with single insertion at apex of left ventricle without cardiopulmonary bypass. The device outflow cannula was positioned across the aortic valve. The international normalized ratio was maintained between 2.0 and 2.5 with warfarin. Hemodynamic, echocardiographic, fluoroscopic, hematologic, and blood chemistry measurements were evaluated.
\end{abstract}

Results: The device was successfully implanted through the left ventricular apex in all 10 animals. The device was operated at 15,000 $\pm 1000 \mathrm{rpm}$ (power consumption, 3.5-6.0 W). The device maintained normal end-organ perfusion with no significant hemolysis $(0-30 \mathrm{mg} / \mathrm{dL})$. There were no pump failures or device-related complications. At autopsy, no abnormalities were seen in endocardium, aortic valve leaflets, or aortic root. There was no evidence of thromboembolism or abnormalities in any peripheral end organs.

Conclusions: We successfully demonstrated feasibility of a novel intraventricular assist device that can be completely implanted through left ventricular apex. This transapical surgical approach eliminates needs for sternotomy, device pocket, cardiopulmonary bypass, ventricular coring, and construction of an outflow graft anastomosis. (J Thorac Cardiovasc Surg 2011;142:668-74)

Mechanical circulatory support has emerged as an important treatment option for patients with end-stage heart failure. Several prospective clinical trials have demonstrated both significant quality of life and survival benefits in patients receiving left ventricular assist devices (LVADs) relative to those treated with optimal medical management alone. ${ }^{1-3}$ Significant clinical experience has now accumulated with the use of the LVAD both as a bridge to transplant and as permanent use or destination therapy. ${ }^{4}$

The incidence of serious adverse events, including infection, bleeding, thromboembolism, and right ventricular dysfunction, associated with LVAD insertion has limited the widespread use of these devices in advanced heart

From the Department of Surgery, ${ }^{\mathrm{a}}$ Cardiovascular Innovation Institute, the Department of Bioengineering, ${ }^{\mathrm{b}}$ and Research Resources Facilities, ${ }^{\mathrm{c}}$ Cardiovascular Innovation Institute, University of Louisville, Louisville, Ky; and HeartWare Inc, ${ }^{d}$ Miami Lakes, Fla.

Supported in part by National Institutes of Health SBIR phase I grant 1 R43 HL103014-01A1.

Disclosures: Authors have nothing to disclose with regard to commercial support.

Received for publication Feb 9, 2010; revisions received Dec 20, 2010; accepted for publication Jan 7, 2011; available ahead of print Feb 14, 2011.

Address for reprints: Mark S. Slaughter, MD, Division of Thoracic and Cardiovascular Surgery, Department of Surgery, University of Louisville, 201 Abraham Flexner Way, Suite 1200, Louisville, KY 40202 (E-mail: mark.slaughter@louisville.edu). $0022-5223 / \$ 36.00$

Copyright (C) 2011 by The American Association for Thoracic Surgery doi:10.1016/j.jtcvs.2011.01.011 failure. ${ }^{1}$ First-generation pulsatile-flow LVADs have been effective in providing mechanical unloading of the heart and restoring normal hemodynamics and end-organ perfusion but are larger, have limited durability because of several moving and contacting parts, require valves to maintain unidirectional flow, and require external venting through a large percutaneous drive line. These limitations have led to the development of smaller continuous-flow LVADs (axial and centrifugal) with fewer moving parts, resulting in higher durability. Further, inflow and outflow conduits do not require valves to maintain antegrade flow. External venting is not needed, allowing use of smaller caliber percutaneous leads. Recent clinical data has demonstrated a favorable risk profile for continuous-flow LVAD relative to first-generation pulsatile designs. ${ }^{5-8}$ Newer generation designs use magnetic coupling and hydrodynamic bearings, allowing a noncontact bearing design that further reduces sources of friction and wear, with the potential to enhance durability and decrease blood activation. ${ }^{8}$

Implantation of one of these continuous-flow devices still requires a major open cardiac surgical procedure, typically requiring median sternotomy, the creation of a preperitoneal or intraperitoneal device pocket, and the use of cardiopulmonary bypass. Long-term implantable LVADs traditionally are placed with inflow to the device from the left ventricular apex and outflow to the ascending aorta, with 


\section{Abbreviations and Acronyms}

LVAD $=$ left ventricular assist device

MVAD $=$ miniature ventricular assist device

construction of an anastomosis between the aorta and outflow conduit. As a result, device therapy has traditionally been offered only to patients in late stages of advanced heart failure. More recently, these devices have been placed retrograde in an indwelling transaortic valve configuration. ${ }^{9-12}$ This newer design enables rapid percutaneous placement without the need for a major surgical procedure. The catheter-based LVADs have been shown to provide adequate mechanical unloading of the ventricle and restore normal end-organ perfusion without damaging aortic valve leaflets or aortic root structures. Current transvalvular devices are designed for short-term support and placed through the femoral vessels, however, rendering the patient immobile and at risk for infection, without the possibility for discharge and outpatient rehabilitation.

The miniature ventricular assist device (MVAD; HeartWare Inc, Miami Lakes, Fla) is a continuous axial-flow pump with a wide-bladed impeller and hydrodynamic fluid bearings. The MVAD was developed from proprietary HeartWare ventricular assist device technology (HeartWare), which is currently in clinical trials. ${ }^{13}$ An intraventricular configuration of the MVAD was developed to allow transapical placement with an outflow cannula advanced antegrade across the aortic valve. This configuration offers the advantages of transapical placement without the use of cardiopulmonary bypass, ease of implantation without the need for ventricular coring or construction of an outflow graft anastomosis, and placement with less invasive surgical approaches (through a subcostal incision or a small thoracotomy). The design and feasibility testing to evaluate hemodynamic efficacy and biocompatibility of the MVAD in acute and long-term large animal models are presented.

\section{MATERIALS AND METHODS \\ Transapical MVAD}

The MVAD is designed for intraventricular implantation with an outflow cannula that crosses the aortic valve. The device can be inserted either through a subcostal incision or through a small left thoracotomy to access the left ventricular apex. The MVAD consists of an anchor section that is attached to the external surface of the myocardium near the apex, a standpipe section that carries the drive cables external to the left ventricle and holds the pump housing off of the internal myocardial surface, a pump housing that contains the MVAD core assembly and a vane diffuser, and an outflow cannula that crosses the aortic valve (Figure 1, top). The outflow cannula tip uses a trilobar diffuser design to provide a radial force component to the outflow, which minimizes the radial movement of the outflow cannula within the aorta. The anchor includes a sewing ring and is designed for fixation of the device and hemostasis. The tip of the trilobar outflow cannula is immediately distal to the aortic root and proximal to the innominate artery (Figure 1, bottom). The MVAD length can be adjusted from 14.5 to $15.5 \mathrm{~cm}$. Its (greatest diameter is $1.75 \mathrm{~cm}$, and weighs $15 \mathrm{~g}$. The priming volume is $15 \mathrm{ml}$, and it is capable of generating flows ranging from 1 to $5 \mathrm{~L} / \mathrm{min}$; however, sustained flow rates less than $2.5 \mathrm{~L} / \mathrm{min}$ may require additional monitoring of anticoagulation status. Operational pump speed ranges between 12,000 and 20,000 rpm. The MVAD flow is adjusted by varying the revolutions per minute set point on the controller through the clinical monitor system, which is similar to the one used for the HeartWare ventricular assist device.

An impeller with dual hydrodynamic bearings on each blade was developed for the MVAD. The impeller is constructed from a single piece of biocompatible, machinable, abrasion-resistant, and magnetizable platinum-based alloy. The single-piece platinum alloy allows each of the bladelike projections to be magnetized as a single, large magnetic pole, which provides a strong electromagnetic coupling to a standard radial motor stator. Further, this strong coupling provides sufficient axial stiffness to keep the impeller properly aligned within the device without the need for mechanical support structures. The impeller radial suspension is accomplished through 2 hydrodynamic bearings, 1 in front and 1 behind the center of mass for enhanced rotor dynamic stability. The simplicity of these impellers built with the platinum alloy eliminates subassembly and the need for abrasion-resistant coatings. The passive impeller suspension system eliminates the requirement for upstream and downstream flow structures.

The MVAD core assembly consists of the impeller, a zirconium ceramic tube to contain the impeller, and a conventional radial motor stator. Zirconium ceramic was used because of its resistance to abrasion damage and minimal eddy current losses. The motor stator is a conventional $12-\mathrm{V}$, 6-slotted design. Conventional radial motor stator design and weld rings allows for simple, hermetic housings in a variety of configurations. The exit cable is $4 \mathrm{~mm}$ in diameter and is covered with velour fabric.

\section{Animal Model}

The MVAD was tested in acute $(\mathrm{n}=4)$ and 1 -week $(\mathrm{n}=2)$ and 30-day $(\mathrm{n}=4)$ long-term experiments in a calf model (male Jersey calves 3-4 months old, $80-100 \mathrm{~kg}$ ). The $80-$ to $100-\mathrm{kg}$ calves were used to match the ventricular apex to aortic valve lengths found in human beings. Animals were allowed to acclimate for 14 days before surgery. This study was conducted in accordance with the Guide for the Care and Use of Laboratory Animals" (National Institutes of Health publication 85-23, 1996, www. nap.edu/catalog/5140.html), the Animal Welfare Act and Regulations, and US Public Health Service Policy on the Humane Care and Use of Laboratory Animals at the University of Louisville, an Association for Assessment and Accreditation of Laboratory Animal Care-accredited program. Use of the animals was approved by the institutional animal care and use committee.

\section{Surgical Technique}

Each animal was anesthetized with $1 \%$ to $5 \%$ isoflurane and $100 \%$ oxygen. An endotracheal tube was inserted to initiate mechanical ventilation. A venous catheter was placed in the marginal ear vein before induction of anesthesia. Tidal volume and respiratory rate were adjusted to maintain arterial oxygen saturation at greater than $93 \%$. Positive end-expiratory pressure was maintained at $6 \mathrm{~cm} \mathrm{H}_{2} \mathrm{O}$. An orogastric tube was placed to evacuate the rumen. The 1-week and 30-day animals received $2.2 \mathrm{mg} / \mathrm{kg}$ flunixin meglumine intravenously and $0.01-\mathrm{mg} / \mathrm{kg}$ buprenorphine or $0.25-$ to $0.5-\mathrm{mg} / \mathrm{kg}$ butorphanol intravenously for analgesia, and $15-$ to $20-\mathrm{mg} / \mathrm{kg}$ cefazolin intravenously before surgery. The calf was placed on the operating table in a right lateral recumbent position to facilitate a left thoracotomy. The skin in the left neck and left thoracic areas was aseptically prepared with chlorhexidine and sterile saline solution or alcohol and sterilely draped. The left jugular furrow was dissected, isolating the left jugular vein and carotid artery. A fluid-filled catheter was placed in 


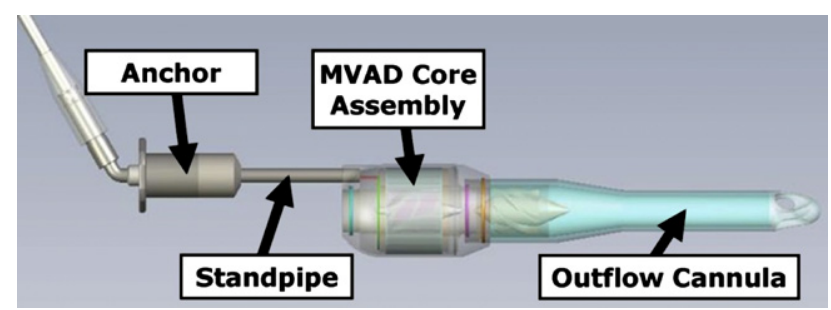

FIGURE 1. Computer aided design model of miniaturized ventricular assist device (MVAD, top). Primary components of this pump are anchor to ventricular apex, standpipe to adjust position of pump inside ventricle and across aortic valve, miniaturized ventricular assist device core assembly, and outflow cannula.

the jugular vein for drug infusion, blood sampling, and venous pressure monitoring. A second fluid-filled catheter was placed into the carotid artery for blood sampling (blood gas values) and arterial pressure monitoring. Lactated Ringer solution was infused to maintain the central venous pressure at 5 to $10 \mathrm{~mm} \mathrm{Hg}$. Fluid boluses of $0.9 \%$ sodium chloride solution were also administered as needed to maintain pressure. Electrocardiogram, arterial line pressure, central venous pressure, pulse oximetry, and body temperature were monitored.

A lidocaine constant-rate infusion $(100 \mathrm{mg} / \mathrm{h})$ was initiated intravenously and titrated for effect to prevent or control cardiac arrhythmias. Boluses of $150 \mathrm{mg}$ amiodarone were given intravenously as needed, also to control cardiac arrhythmias. With the animal in a surgical plane of anesthesia (as determined by the loss of palpebral reflex, ventral pupil fixation, and lack of response to painful stimuli), $0.1-\mathrm{mg} / \mathrm{kg}$ pancuronium bromide or $30 \mathrm{mg}$ succinylcholine (INN suxamethonium chloride) was administered intravenously before initiation of left thoracotomy incision. Calves were monitored continuously during anesthesia, and body temperature was maintained with a warm water or air-circulating blanket. A left thoracotomy was performed at the 5th intercostal space to provide access and exposure of the pulmonary artery, left ventricular apex, and descending thoracic aorta.

The MVAD was filled with normal saline solution. Heparin (200-300 U/kg by intravenous central line) was administered. From 6 to 8 pledgeted sutures were fixed to the left ventricular apex. After a small incision had been made in the left ventricular apex, insertion and fixation of the MVAD was completed without a need for bypass. Echocardiography and fluoroscopy were performed on the calf to verify anatomic positioning of the MVAD inside the aorta and to position the outflow cannula across the aortic valve. If needed, the length of the standpipe was adjusted to ensure proper anatomic fit. The chronic animals were anticoagulated with heparin for 72 hours after device implantation (activated coagulation time target, 200-300 seconds) and then with warfarin to maintain an international normalized ratio of 2.0 to 3.0. Postoperatively, long-term animals also received $15-$ to $20-\mathrm{mg} / \mathrm{kg}$ cefazolin twice daily intravenously for 7 days. Analgesia consisted of 1.1 - to $2.2-\mathrm{mg} / \mathrm{kg}$ flunixin meglumine intravenously 1 to 2 times daily for 3 days and $0.01-\mathrm{mg} / \mathrm{kg}$ buprenorphine twice daily or 0.25 - to $0.5-\mathrm{mg} / \mathrm{kg}$ butorphanol intravenously every 4 hours for 2 days.

\section{Measurements and Analyses}

In the acute calf experiments, a high-fidelity pressure catheter (Millar Instruments, Houston, Tex) was used to measure left atrial pressure. A high-fidelity dual pressure-volume conductance catheter (Millar Instruments) was used for simultaneous measurement of left ventricular pressure and volume and aortic pressure. Transit-time flow probes (Transonics, Ithaca, NY) were used for measurement of aortic flow. All transducers were precalibrated and postcalibrated against known standards to ensure measurement accuracy. Hemodynamic data were signal conditioned and analog to digital converted at $400-\mathrm{Hz}$ sampling rate for digital analysis with a real-time good laboratory practice-compliant data-acquisition system. ${ }^{14}$ Hemodynamic waveforms were recorded during (1) baseline, (2) partial MVAD support, and (3) full MVAD support. Baseline measurements were repeated between test conditions to ensure that steady state baseline was maintained. Characterization of hemodynamic parameter values was calculated with a Hemodynamic Evaluation and Assessment Research Tool (HEART) program developed in Matlab (MathWorks, Natick, Mass). ${ }^{15}$ Pressure, flow, and volume waveforms were used to calculate landmark cardiovascular function parameters (heart rate, stroke volume, cardiac output, and mean, systolic, and diastolic pressures) on a beat-to-beat basis, with all beats in each data set averaged to obtain a single representative mean value for each parameter. Left ventricular pressure-volume loops were constructed by plotting ventricular pressure against ventricular volume, with each loop representing 1 complete cardiac cycle (1 beat). Pressure-volume loops were used to calculate external work (area inside pressure-volume loop) and to determine ventricular filling and ejection pressures and changes in ventricular enddiastolic, end-systolic, and stroke volumes. In the long-term animal experiments, a flow probe was placed on the pulmonary artery (cardiac output), and an arterial fluid-filled pressure line (mean arterial pressure) was placed.

\section{Echocardiography}

Echocardiography was performed before, during, and after surgery to assess cardiac function, blood flow, and device placement. A Philips iE33 Echocardiography System with S8-3 transducer (Koninklijke Philips Electronics NV, Amsterdam, The Netherlands) was used to obtain M-mode and Doppler echocardiographic recordings. The transducer was covered by a sterile latex sheath, with ultrasound gel inside the sheath to facilitate acoustic transmission. Sterilized warm ultrasound gel was also placed on the epicardium during imaging. Parasternal and apical views were used to obtain images. Left ventricular end-diastolic and end-systolic volumes and left ventricular ejection fraction (systolic function) were determined from the apical 4-chamber view with the modified Simpson technique (summation of discs). Left ventricular diastolic performance was assessed by tissue Doppler imaging at the level of the mitral annulus. Right ventricular size was indexed by the planimetered right ventricular end-diastolic and end-systolic areas and right ventricular systolic function by the fractional area change. The parasternal view was used to measure left ventricular end-diastolic and end-systolic diameters and wall thicknesses orthogonal to the long-axis of the left ventricle and to evaluate aortic valve opening and MVAD placement. Regional wall thickening was assessed at the anteroseptum and posterior wall. Color Doppler was used to detect and quantify aortic regurgitation with the MVAD outflow cannula positioned across the aortic valve (1-4+ severity scale).

\section{Fluoroscopy}

Fluoroscopy was performed to evaluate blood flow and anatomic positioning of the MVAD in cardiac catheterization laboratory (GE Innova 3100 All-Digital Cardiovascular Imaging System; GE Healthcare, a division of General Electric Company, Chalfont St Giles, United Kingdom). A cutdown to the right carotid artery was performed, and a vascular sheath was placed in the carotid artery. An angiography catheter was placed in the left ventricle for injection of radiopaque dye $(50-100 \mathrm{~mL}$, repeated 3-5 times) for flow visualization during fluoroscopy.

\section{Hematology and Blood Chemistry}

Venous blood samples $(5 \mathrm{~mL})$ were drawn to measure complete blood cell count (Hemavet 950; CDC Technologies, Inc, Oxford, Conn), serum chemistries (Jewish Hospital, Louisville, Ky) and plasma free hemoglobin (HemoCue AB, Ängelholm, Sweden). During the acute animal experiments, venous blood samples were withdrawn before MVAD implantation (baseline), hourly during MVAD support, and after MVAD support 


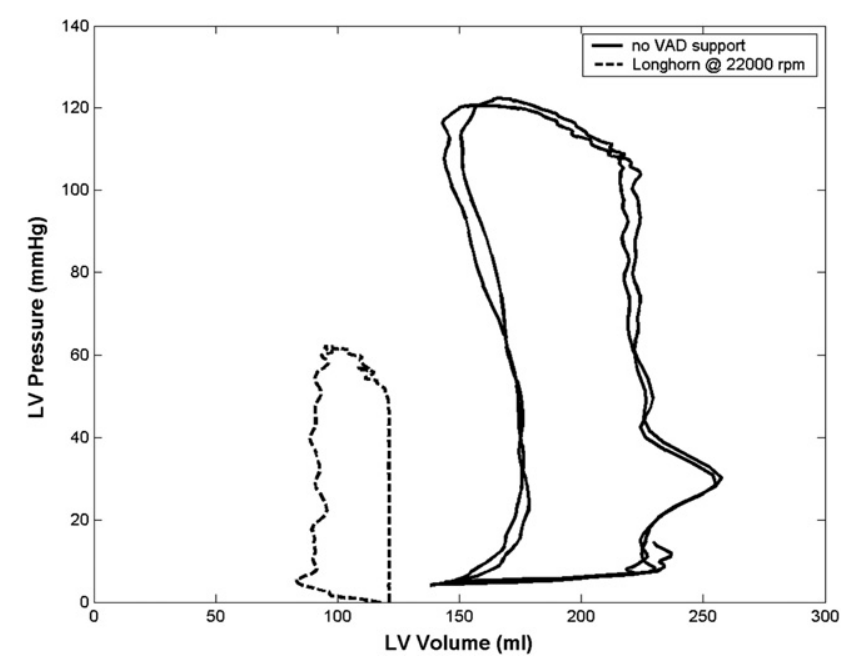

FIGURE 2. Left ventricular $(L V)$ pressure-volume loops demonstrating effective mechanical unloading of ventricle with device support, as evidenced by leftward shift of PV loop and reduced ventricular filling and ejection pressures during miniaturized ventricular assist device $(V A D)$ support.

(baseline). During the long-term animal experiments, venous blood samples were collected before MVAD implantation (baseline) and after MVAD implantation (every 12 hours for the first 2 days, daily from days $3-7$, and weekly thereafter until the end of study) to analyze for temporal changes in biochemical markers and quantify plasma free hemoglobin.

\section{Histopathology}

At the completion of the experiment, the MVAD was visually inspected for evidence of clots, fractures, or other defects and photographed. End organs were examined at necropsy for evidence of embolization and photographed. Histopathologic analyses were conducted on the aortic valve and aortic root to ensure that the MVAD did not cause any injury to the aortic valve or aorta. Aortic valve and vascular tissue blocks were sectioned at 5 - $\mu \mathrm{m}$ thick and prepared for staining with hematoxylin and eosin for visualization of histopathologic changes under light microscopy.

\section{RESULTS}

\section{Acute Large Animal Study}

The MVAD was successfully implanted through a limited left thoracotomy, and the transapical placement of the MVAD outflow cannula was confirmed by echocardiography in all 4 acute animal experiments. There were no complications related to device implantation or device performance, and there was no apical bleeding. The MVAD reduced ventricular workload, as evidenced by lower systolic and end-diastolic left ventricular pressures and smaller ventricular volumes (Figure 2 and Table 1). Further, MVAD support increased mean, systolic, and diastolic aortic pressures and mean aortic flows (Table 1). Aortic pressure pulsatility diminished with increasing MVAD support (Figure 3). Collectively, these data demonstrated that the MVAD effectively mechanically unloaded the native ventricle. A careful necropsy of the calves demonstrated no evidence of embolization in the brain, kidneys, spleen, or heart; however, thrombus formation was observed at the junction between the pump and the outflow cannula in 1 of the 4 experiments. A computational fluid dynamics study conducted after the acute studies revealed an area of stasis at the junction between the device and the outflow cannula. The outflow cannula and cannula junction were redesigned to eliminate this thrombus formation.

\section{Long-Term Large Animal Study}

MVADs were successfully implanted in all 6 long-term animals. Average speed (in rpm) and power consumption (in watts) for the MVAD were within operational tolerances throughout the duration of long-term support. There were no device or controller system failures with the MVAD during the study period. There were no apical bleeding issues in any of the long-term animal experiments. The calves were electively killed at 1 week $(n=2)$ or 30 days $(n=3)$ after implant with $0.02-\mathrm{mg} / \mathrm{kg}$ xylazine intravenously followed by $1 \mathrm{~mL} / 10 \mathrm{lb}$ body weight Beuthanasia-D Special (Intervet/Schering-Plough Animal Health; Summit, NJ). One 30-day calf was electively killed at 14 days because of pulmonary infection and ongoing respiratory distress not related to MVAD support, as documented by necropsy. Echocardiography and fluoroscopy demonstrated stable device position of the MVAD within the ventricular cavity (Figure 4). No aortic insufficiency was observed during fluoroscopy or echocardiography. There were no changes in hepatic function (alkaline phosphates, alanine aminotransferase) or renal function (creatinine) from baseline, suggesting that end-organ function was preserved. Plasma

TABLE 1. Mean aortic flow, peak left ventricular pressure, left ventricular end diastolic pressure, left ventricular external work, positive and negative rates of change in left ventricular pressure, mean aortic pressure, systolic aortic pressure, and diastolic aortic pressure during baseline (no miniaturized ventricular assist device support), and with miniaturized ventricular assist device support

\begin{tabular}{lccccccccc}
\hline Condition & $\begin{array}{c}\text { AoFm } \\
(\mathbf{L} / \mathbf{m i n})\end{array}$ & $\begin{array}{c}\text { LVPsys } \\
(\mathbf{m m} \mathbf{H g})\end{array}$ & $\begin{array}{c}\text { LVPed } \\
(\mathbf{m m} \mathbf{H g})\end{array}$ & $\begin{array}{c}\text { LVEW } \\
(\mathbf{m m} \mathbf{H g} \cdot \mathbf{m L})\end{array}$ & $\begin{array}{c}+\mathbf{d P} / \mathbf{d t} \\
(\mathbf{m m} \mathbf{H g} / \mathbf{s})\end{array}$ & $\begin{array}{c}-\mathbf{d P} / \mathbf{d t} \\
(\mathbf{m m} \mathbf{H g} / \mathbf{s})\end{array}$ & $\begin{array}{c}\text { AoPm } \\
(\mathbf{m m} \mathbf{H g})\end{array}$ & $\begin{array}{c}\text { AoPsys } \\
(\mathbf{m m} \mathbf{H g})\end{array}$ & $\begin{array}{c}\text { AoPdia } \\
(\mathbf{m m} \mathbf{H g})\end{array}$ \\
\hline Baseline & $5.7 \pm 1.5$ & $91 \pm 6$ & $12 \pm 0.4$ & $6237 \pm 109$ & $791 \pm 40$ & $-1187 \pm 3$ & $81 \pm 6$ & $91 \pm 2$ & $73 \pm 8$ \\
MVAD support & $6.3 \pm 1.7$ & $70 \pm 5$ & $6 \pm 3.3$ & $3299 \pm 1212$ & $628 \pm 25$ & $-795 \pm 138$ & $85 \pm 1$ & $96 \pm 6$ & $76 \pm 6$ \\
\hline
\end{tabular}

These data demonstrate unloading of native left ventricle and improvement in flow with miniaturized ventricular assist device support. AoFm, Mean aortic flow; $L V P$ sys, peak (systolic) left ventricular pressure; $L V P e d$, left ventricular end-diastolic pressure; $L V E W$, left ventricular external work; $+d P / d t$, positive rate of change in left ventricular pressure; $-d P / d t$, negative rate of change in left ventricular pressure; $A o P m$, mean aortic pressure; AoPsys, systolic aortic pressure; AoPdia, diastolic aortic pressure; $M V A D$, miniaturized ventricular assist device. 

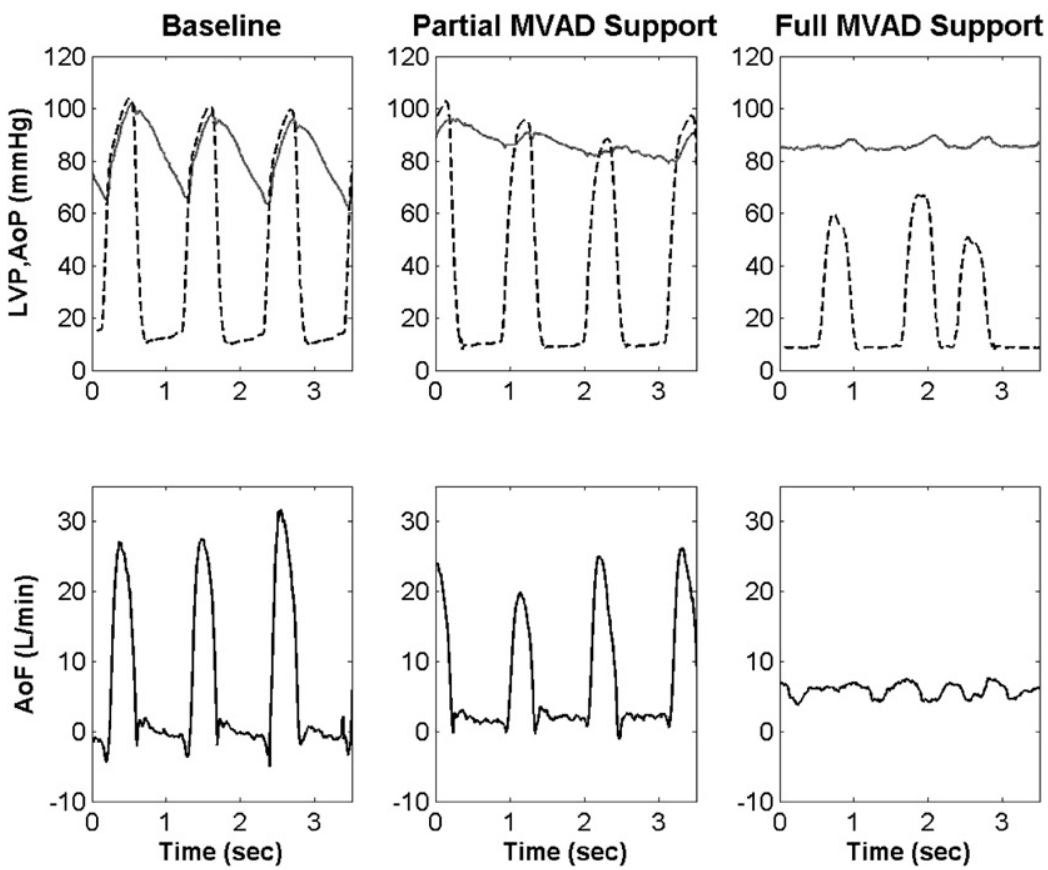

FIGURE 3. Aortic pressure ( $A o P)$, left ventricular pressure ( $L V P$, dashed), and aortic flow $(A o F)$ waveforms during baseline, partial miniaturized ventricular assist device (MVAD) support, and full miniaturized ventricular assist device support. These data demonstrate that miniaturized ventricular assist device unloads native ventricle similar to clinical ventricular assist devices.
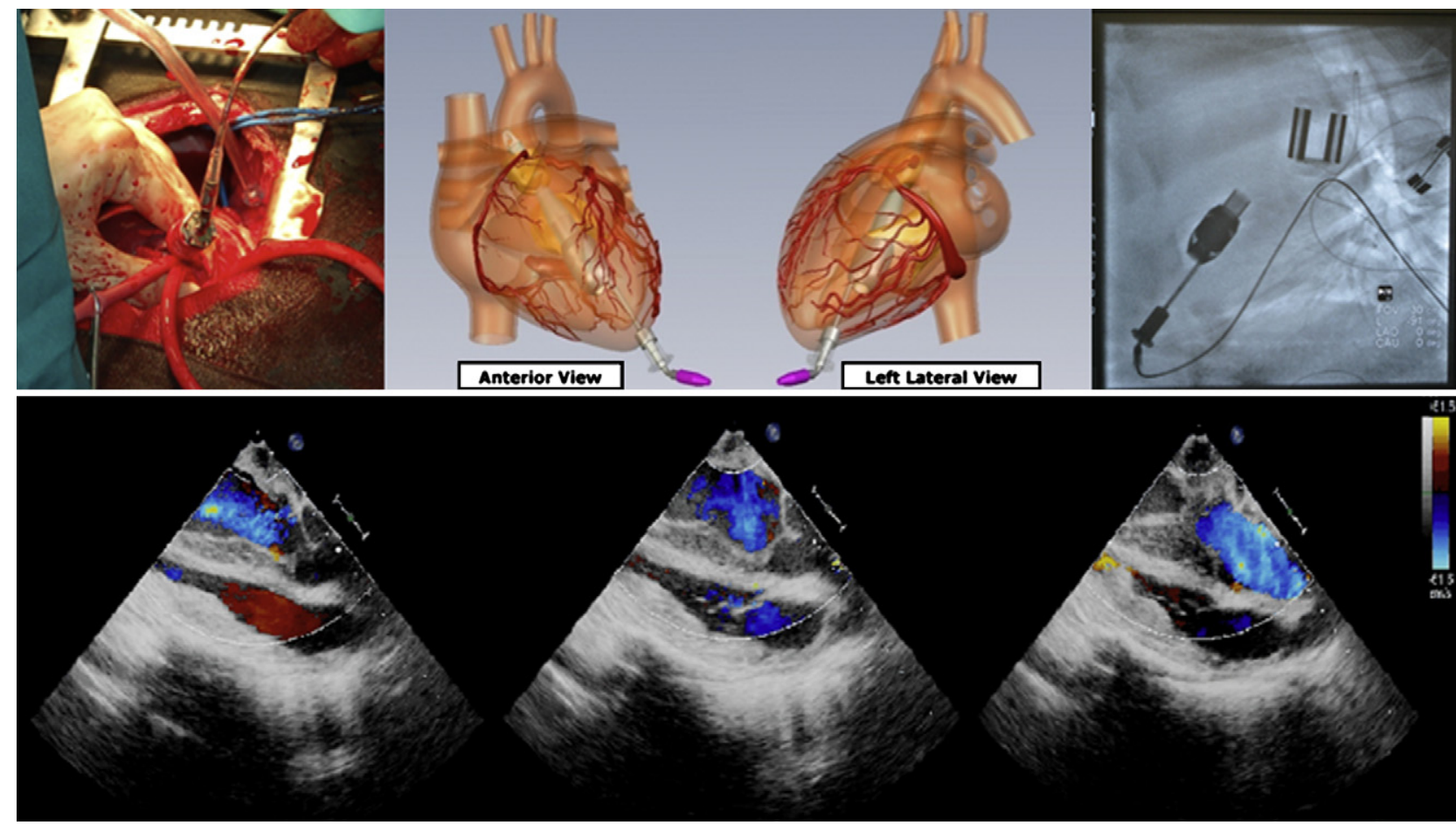

FIGURE 4. Apex of left ventricle was accessed via thoracotomy. Miniaturized ventricular assist device was inserted through apex of left ventricle (top left). Graphic illustration of implanted positioning of miniaturized ventricular assist device demonstrating transapical placement (top center). Fluoroscopy shows good anatomic placement of miniaturized ventricular assist device in calf (top right). Echocardiographic color Doppler images demonstrate minimal aortic regurgitation and adequate coaptation of aortic valve with transapical outflow cannula placement (bottom). 


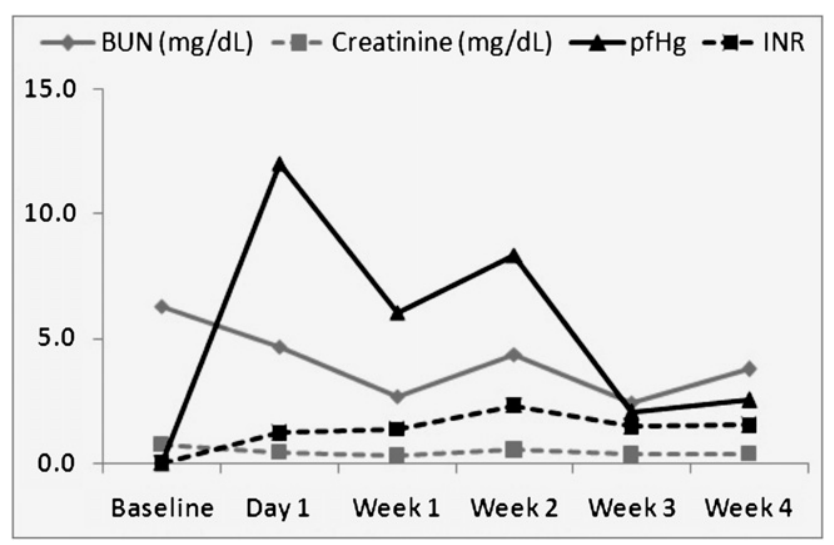

FIGURE 5. Average blood urea nitrogen $(B U N)$, creatinine, plasma free hemoglobin $(p f H g)$, and international normalized ratio (INR) values for all animals $(\mathrm{n}=4)$ were within clinically accepted thresholds throughout 30-day study period. These results demonstrate that miniaturized ventricular assist device carries minimal-device related hemolysis.

free hemoglobin, blood urea nitrogen, creatinine, and international normalized ratio values were normal at all measured time points (Figure 5). At necropsy, all explanted MVADs were free of thrombus formation. Histopathologic examination demonstrated no abnormalities within the endocardium, mitral and aortic valve leaflets, or aortic root (Figure 6). There was no evidence of thromboembolism or abnormalities in any peripheral end-organ system.

\section{DISCUSSION}

The transapical HeartWare MVAD has a number of potential advantages relative to second-generation devices currently in use today. The MVAD is significantly smaller and requires a single insertion through the apex of the left ventricle with the distal end of the device crossing the aortic valve. Earlier generations of implantable LVADs have not been specifically designed for myocardial recovery or partial support but rather to serve as a therapeutic bridge to heart transplant or as destination therapy. ${ }^{16}$ Patients in New York Heart Association class III or early class IV heart failure with preserved end-organ function may not require complete mechanical cardiac replacement by LVAD and may have a higher likelihood of recovery. ${ }^{17}$ Data from the Interagency Registry for Mechanically Assisted Circulatory Support demonstrate improved clinical outcomes when device therapy is implemented at earlier stages of heart failure. ${ }^{18,19}$ The unique wide-bladed design of this axial-flow pump with incorporated hydrodynamic thrust bearings provides limited contact between the stator and the rotor. This has the potential to limit the degradation of rotor components and to minimize the potential for thrombosis. The rotor design also incorporates a rigid magnetic coupling, which may lead to reduced cellular trauma and heat generation, preventing activation of circulating blood elements and minimizing hemolysis. Clinically, this may translate into reductions in perioperative and postoperative bleeding complications and the associated risks of transfusion.

The MVAD outflow cannula is positioned across the aortic valve, and the device can be implanted off pump, without a standard median sternotomy or ventricular coring, thus using less invasive surgical techniques. The echocardiographic and fluoroscopic data from 30-day implants indicated that the aortic valve leaflets coapted around the outflow cannula, and there were no significant retrograde flows observed at the aortic valve. Further, no histopathologic changes were identified in the aortic valve leaflets. Longer-term implant (90 day) and recovery data need to be completed for further investigation the potential effects and clinical implications. In light of the current clinical data suggesting that even mild aortic insufficiency at the time of device implant potentially worsens during the support period in the continuous-flow pump population, further evaluation is needed to determine whether aortic insufficiency would be a contraindication for this new pump. The MVAD tested in the bovine model would be appropriate for patients with a body surface area of $1.5 \mathrm{~m}^{2}$ or larger. As a result of this feasibility study, variable-length cannulas will be integrated into the MVAD design to accommodate smaller, shorter intraventricular lengths. Further, the device may be operated as either a partial support or a complete
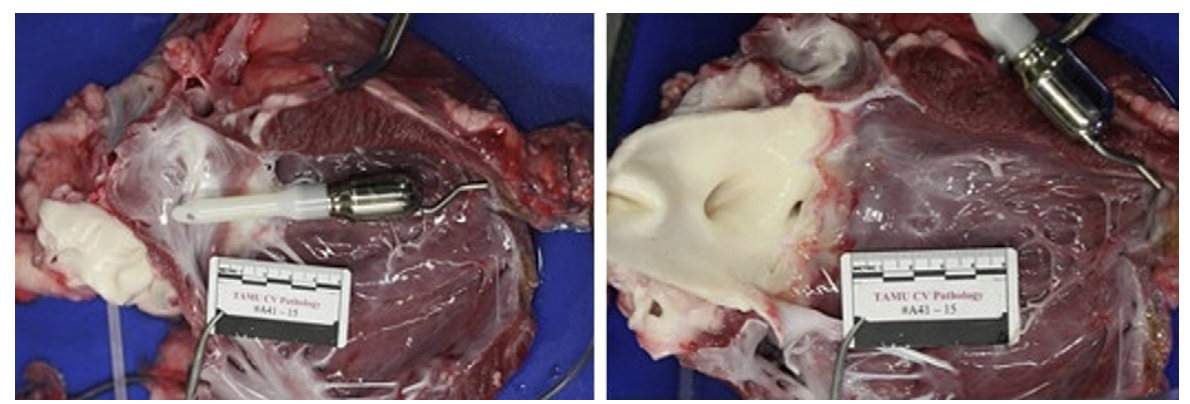

FIGURE 6. Heart and aortic valve after animal was electively killed 30 days after miniaturized ventricular assist device implantation. Device was entirely free of thrombi (left) in all long-term animals, and there was no damage to aortic valve (right), demonstrating feasibility of long-term hemocompatibility with miniaturized ventricular assist device. 
support device, as dictated by the clinical presentation and myocardial condition of the patient. The transapical MVAD implantation technique eliminates the need for a median sternotomy, the creation of a device pocket, the use of cardiopulmonary bypass, ventricular coring, and the construction of an outflow graft anastomosis. This could potentially result in less surgical trauma, reduced operative time, and reduced transfusion requirements, which could be another incremental step forward to reduce adverse events associated with current continuous-flow LVAD support.

\section{CONCLUSIONS}

The acute and long-term animal experiments clearly demonstrate adequate anatomic fit, hemodynamic efficacy, and potential long-term biocompatibility of the transapical MVAD. Further studies will focus on the refinement of the system controller, engineering of control strategies, and longer-term 90-day studies.

We acknowledge and thank Cary Woolard, Mary Anne Hauck, Laura and Karen Lott, and the University of Louisville Research Resources Facilities veterinary staff for their assistance.

\section{References}

1. Rose EA, Gelijns AC, Moskowitz AJ, Heitjan DF, Stevenson LW, Dembitsky W, et al. Long-term mechanical left ventricular assistance for end-stage heart failure. N Engl J Med. 2001;345:1435-43.

2. Rogers JG, Butler J, Lansman SL, Gass A, Portner PM, Pasque MK, et al. Chronic mechanical circulatory support for inotrope-dependent heart failure patients who are not transplant candidates: results of the INTrEPID Trial. J Am Coll Cardiol. 2007;50:741-7.

3. Pae WE, Connell JM, Adelowo A, Boehmer JP, Korfer R, El-Banayosy A, et al. Does total implantability reduce infection with the use of a left ventricular assist device? The LionHeart experience in Europe. J Heart Lung Transplant. 2007;26: 219-29.

4. Slaughter MS, Rogers JG, Milano CA, Russell SD, Conte JV, Feldman D, Sun B, et al. Advanced heart failure treated with continuous-flow left ventricular assist device. N Engl J Med. 2009;361:2282-5.
5. Miller LW, Pagani FD, Russell SD, John R, Boyle AJ, Aaronson KD, et al. Use of a continuous-flow device in patients awaiting heart transplantation. $N$ Engl J Med. 2007;357:885-96.

6. John R, Kamdar F, Liao K, Colvin-Adams M, Boyle A, Joyce L. Improved survival and decreasing incidence of adverse events with the HeartMate II left ventricular assist device as bridge-to-transplant therapy. Ann Thorac Surg. 2008;86: 1227-35.

7. Patel ND, Weiss ES, Schaffer J, Ullrich SL, Rivard DC, Shah AS, et al. Right heart dysfunction after left ventricular assist device implantation: a comparison of the pulsatile HeartMate I and axial-flow HeartMate II devices. Ann Thorac Surg. 2008;86:832-40

8. Pagani FD. Continuous-flow rotary left ventricular assist devices with "3rd generation" design. Semin Thorac Cardiovasc Surg. 2008;20:255-63.

9. Dixon S, Henriques JP, Mauri L, Sjauw K, Civitello A, Kar B, et al. A prospective feasibility trial investigating the use of the Impella 2.5 system in patients undergoing high-risk percutaneous coronary intervention (The PROTECT I Trial): initial U.S. experience. JACC Cardiovasc Interv. 2009;2:91-6.

10. Meyns B, Dens J, Sergeant P, Herijgers P, Daenen W, Flameng W. Initial experiences with the Impella device in patients with cardiogenic shock-Impella support for cardiogenic shock. Thorac Cardiovasc Surg. 2003;51:312-7.

11. Vercaemst L, Vandezande E, Janssens P, Yvan T, Peter D, Meyns B. Impella: a miniaturized cardiac support system in an era of minimal invasive cardiac surgery. J Extra Corpor Technol. 2002;34:92-100.

12. Siess T, Nix C, Menzler F. From a lab type to a product: a retrospective view on Impella's assist technology. Artif Organs. 2001;25:414-21.

13. Slaughter MS, Sobieski MA 2nd, Tamez D, Horrell T, Graham J, Pappas PS, et al. HeartWare miniature axial-flow ventricular assist device: design and initial feasibility test. Tex Heart Inst J. 2009;36:12-6.

14. Koenig SC, Woolard C, Drew G, Unger L, Gillars K, Ewert D, et al. Integrated data acquisition system for medical device testing and physiology research in compliance with good laboratory practices. Biomed Instrum Technol. 2004;38: 229-40.

15. Schroeder MJ, Perrrault B, Ewert DL, Koenig SC. HEART: an automated beatto-beat cardiovascular analysis package using Matlab. Comput Biol Med. 2004; 34:371-88.

16. Holman WL, Bourge RC, Spruell RD, Murrah CP, McGiffin DC, Kirklin JK. Ventricular assist devices as a bridge to cardiac transplantation. A prelude to destination therapy. Ann Surgery. 1997;225:695-706.

17. Morley D, Litwak K, Ferber P, Spence P, Dowling R, Meyns B, et al. Hemodynamic effects of partial ventricular support in chronic heart failure: results of simulation validated with in vivo data. J Thorac Cardiovasc Surg. 2007;133:21-8.

18. Holman WL, Pae WE, Teutenberg JJ, Acker MA, Naftel DC, Sun BC, et al. INTERMACS: interval analysis of registry data. J Am Coll Surg. 2009;208: 755-62.

19. Kirklin JK, Naftel DC, Stevenson LW, Kormos RL, Pagani FD, Miller MA, et al. INTERMACS database for durable devices for circulatory support: first annual report. J Heart Lung Transplant. 2008;27:1065-72. 